\title{
Tecnologias e Educação Ambiental: Uma Cartilha Interativa Digital para Aprendizagem Sobre Lixo Eletrônico
}

\author{
Helder Gabriel da Silva Pereira \\ Instituto Federal de Mato Grosso do Sul - IFMS \\ helder.pereira@estudante.ifms.edu.br \\ Isabella Natal da Silva \\ Instituto Federal de Mato Grosso do Sul - IFMS \\ isabella.silva@estudante.ifms.edu.br
}

\author{
Sheron Orteney \\ Instituto Federal de Mato Grosso do Sul - IFMS \\ sheron.orteney@estudante.ifms.edu.br \\ Claudio Zarate Sanavria \\ Instituto Federal de Mato Grosso do Sul - IFMS \\ claudio.sanavria@ifms.edu.br
}

\begin{abstract}
This paper describes the results of a project whose general objective was to develop an interactive digital booklet with an educational character that addressed aspects related to technological waste and the environment. To achieve the proposed general objective, the following specific objectives were established: 1) To develop material that would bring educational content in line with current discussions and knowledge about electronic waste and its impacts on the environment; 2) To offer an interdisciplinary and interactive environment that would allow the user to go deeper into the themes, according to their interests and according to the level of information consistent with their age and education; 3) Allow the population to have access to the material free of charge and with possibilities to contribute to its constant improvement. The developed product presents informative contents, interspersed with pastimes for its fixation, mediated by mascots specially developed for the material. The results point to an acceptance by the target audience (high school students) and show an interest in the theme from its handling.
\end{abstract}

\section{KEY WORDS}

Environmental Education, Electronic Waste, Educational Booklet.

\section{INTRODUÇÃO}

O grande avanço tecnológico atualmente vivenciado no mundo todo trouxe grandes consequências em termos ambientais. O fácil acesso econômico e a rápida obsolescência dos equipamentos criaram uma rotina de descarte que contribui para um preocupante acúmulo de resíduos tecnológicos e acaba por contaminar o ambiente. Salvo os poucos brasileiros que vivem em cidades onde há coleta seletiva, os demais têm duas opções: guardar o material tóxico dentro de casa ou jogá-lo no lixo e torcer para não contaminar o meio ambiente [1].

Essa demanda tem aumentado significativamente o volume de resíduos eletrônicos e, como consequência, o descarte desse material acaba sendo em lixos comuns ou em lixões onde são queimados, sem precauções e consciência de que o eventual processo pode acarretar danos ao meio ambiente e agravos à saúde [2].

No mesmo ritmo do acúmulo de resíduos está o gasto de recursos naturais esgotáveis. Dessa forma, é necessário que haja um uso consciente das ferramentas físicas da computação em práticas menos prejudiciais.

Dados da Organização das Nações Unidas (ONU) apontam um recorde de 53,6 milhões de toneladas métricas (Mt) de lixo eletrônico gerado em todo o mundo em 2019, um aumento de $21 \%$ em apenas cinco anos, de acordo com o relatório Global E-waste Monitor. A Ásia gerou o maior volume de lixo eletrônico em 2019 - cerca de 24,9 Mt, seguida pelas Américas (13,1 Mt) e Europa (12 $\mathrm{Mt}$ ), enquanto a África e a Oceania geraram 2,9 Mt e 0,7 Mt, respectivamente [3].

Esse tipo de resíduo não deve ser jogado junto com o lixo orgânico. Os eletrônicos possuem metais pesados altamente tóxicos, como mercúrio, cádmio, berílio e chumbo que, liberados em um aterro, podem contaminar o lençol freático e poluem o ar se forem queimados. Além disso, muitos componentes eletrônicos podem ser reciclados, aproveitando os minerais presentes neles e diminuindo a pressão por mineração, uma atividade econômica com potencial para causar grande dano ao meio ambiente [4].

É necessário que o ser humano não tenha um contato direto com os resíduos de lixo eletrônico para não se contaminar e, para evitar os tipos de contaminações presentes, é preciso que o material seja descartado de forma correta [5]. Ainda mais que os danos causados ao meio ambiente, inclusive ao homem, em muitas vezes mostramse irreversíveis e o contato com substâncias tóxicas decorrente do manejo desses equipamentos contribui para isso [6].

Apesar de existirem diversos ambientes de aplicabilidade da TI Verde, ainda é baixa a adesão às suas práticas. Entretanto, o fato de tais iniciativas agregarem valor que futuramente não poderão ser dispensados torna a TI Verde uma prioridade nas grandes e pequenas empresas [7]. Impulsionados pela regulamentação, os sistemas de coleta formal também vêm ganhando mais importância, assim como ações individuais ou coletivas. Quantidades muito grandes de lixo eletrônico ainda são administradas pelo setor informal ou, na melhor das hipóteses, armazenados em porões [3]. O setor informal predomina na América Latina, mas poucos países, 
como Brasil e Chile, estão abordando ativamente o seu papel em relação à gestão do lixo eletrônico. O reconhecimento, a regulamentação e a integração do seu trabalho nesta área são grandes desafios da região [3].

Mesmo que se pense que, inicialmente, as iniciativas quanto ao lixo eletrônico devam partir das empresas que produzem as tecnologias, é importante destacar aqui a importância de se iniciarem campanhas que promovam a consciência ambiental no cidadão comum. Assim, pensando no município de Nova Andradina e região, são poucas as iniciativas dessa natureza.

Mais do que o simples descarte dos resíduos tecnológicos, entende-se como primordial a conscientização quanto ao consumo e o despertar de uma ação crítica diante da obsolescência programada e perceptiva estimulada pela mídia à população. Independente da visão do ser humano sobre a forma de reciclagem, ou seja, visando uma perspectiva de desafio ou oportunidade de lucro, é necessário que haja uma conscientização e posterior mudança de hábitos relacionados à toda cadeia de fabricação, aquisição e descarte de aparelhos eletroeletrônicos [6].

Tal contexto de desenvolvimento tecnológico e consequente produção de resíduos faz com o que projeto cujos resultados serão aqui descritos tenha um duplo valor: de um lado, traz para a população e, em especial, à comunidade estudantil, a possibilidade de compreender o percurso histórico das tecnologias digitais; e, por outro, permitirá um debate mais consciente quanto ao consumo e reaproveitamento dos recursos tecnológicos.

Visando o início de um trabalho de conscientização junto à comunidade de Nova Andradina, Mato Grosso do Sul, o IFMS realiza desde 2012 - em parceria com órgãos públicos municipais - uma campanha de coleta de resíduos tecnológicos. Em média são coletadas seis toneladas de material por ano, sendo parte dele incorporado por diversos projetos de extensão e pesquisa do IFMS Campus Nova Andradina.

Neste contexto, como mais uma ação ambiental do IFMS Campus Nova Andradina, o presente projeto de extensão propôs, elaborou e distribuiu uma cartilha interativa digital apresentando informações sobre o lixo eletrônico, sua procedência e impactos ao meio ambiente, de modo lúdico e educativo, a partir do levantamento prévio de pesquisas relacionadas e a construção de um conteúdo coeso e em consonância com as atuais necessidades inerentes à Educação Ambiental.

\section{OBJETIVOS}

O projeto aqui descrito teve por objetivo geral desenvolver uma cartilha interativa digital com caráter educativo que abordasse aspectos relacionados aos resíduos tecnológicos e o meio ambiente. Para o alcance o objetivo geral proposto, foram estabelecidos os seguintes objetivos específicos: 1) Desenvolver um material que trouxesse um conteúdo educativo em consonância com as atuais discussões e conhecimentos acerca do lixo eletrônico e seus impactos ao meio ambiente; 2) Oferecer um ambiente interdisciplinar e interativo que permitisse ao usuário maior aprofundamento nos temas, conforme seus interesses e de acordo com o nível de informação coerente com sua idade e escolaridade; 3) Permitir que a população tivesse acesso ao material de modo gratuito e com possibilidades de contribuição para seu aprimoramento constante.

\section{PERCURSO METODOLÓGICO}

Para o desenvolvimento do presente projeto, primeiramente foi necessário o levantamento prévio do estado da arte acerca do lixo eletrônico, buscando informações que fossem relevantes sob o ponto de vista da Educação Ambiental. Para isso, foram selecionados artigos em periódicos, além de livros e revistas especializados.

Uma vez levantadas as informações, a próxima etapa consistiu na elaboração de um roteiro para a cartilha, com criação de tópicos e personagens mascotes que auxiliassem na leitura e no acesso aos dados. Foi elaborado um protótipo de baixa fidelidade, analisado por um grupo focal de potenciais leitores - representativo do público-alvo do projeto - a fim de que ajustes fossem realizados no roteiro. Antes da apresentação do protótipo, foi aplicado um questionário ao grupo, buscando levantar dados sobre suas expectativas acerca do material.

A partir da aprovação do protótipo foi desenvolvida a versão final da cartilha. Inicialmente pretendia-se utilizar tecnologias de desenvolvimento Web para que o produto pudesse ser disponibilizado de modo online aos seus usuários finais. Entretanto, a pandemia Covid-19 impactou consideravelmente no desenvolvimento do projeto, sendo redefinida a mídia para um arquivo pdf. Essa foi a maneira encontrada para viabilizar a continuidade dos trabalhos de modo remoto e em tempo hábil.

Após a disponibilização da versão final da cartilha, um novo questionário foi aplicado para que se se analisassem a receptividade do produto e os principais pontos de melhoria para futuras versões.

Nesse contexto, a análise dos resultados do projeto aqui descrito consistiu numa verificação da versão final e do cruzamento dos dados coletados nos dois questionários.

\section{DESCRIÇÃO DO PRODUTO}

A cartilha possui conteúdo distribuído em 16 páginas e organizados de modo a intercalar informação com passatempos para fixação dos conteúdos abordados. A ideia foi desenvolver um produto acessível a estudantes da fase final do Ensino Fundamental e Ensino Médio, pois traz informações relacionadas a conteúdos de Química, assim como alguns dados estatísticos.

Foram definidos três personagens como mascotes, a fim de direcionar a leitura e tornar o material mais atrativo para os seus leitores. Fazendo uma relação com o fato do lixo eletrônico representar sempre algo mais "velho" ou com um grande tempo de uso, um dos personagens consiste em um "vovô disquete", representando um pouco os materiais obsoletos, ou seja, que não possuem mais possibilidades de uso com os atuais recursos tecnológicos disponíveis. Ao mesmo tempo, os outros personagens representam os "netinhos pendrives", fazendo uma analogia aos 
dispositivos de armazenamento mais recentes, porém com um grande potencial de descarte, considerando sua fragilidade e, principalmente, o lançamento de dispositivos com capacidade de armazenamento cada vez maior. A Figura 1 ilustra a capa da cartilha.

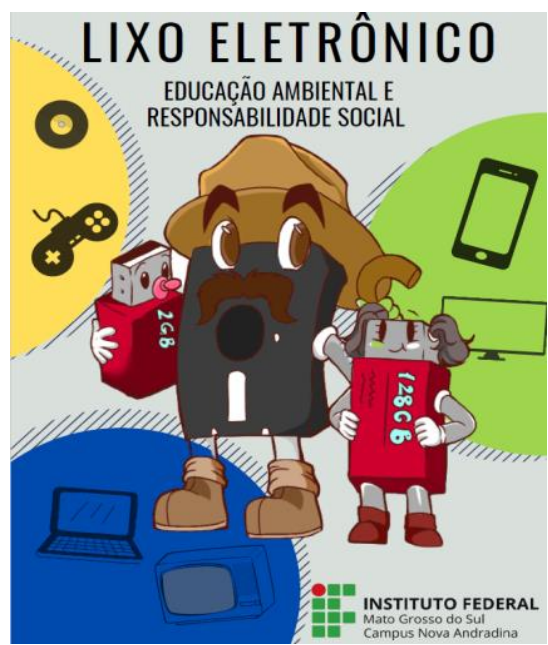

Figura 1: Capa da cartilha.

A cartilha traz, inicialmente, conteúdo introdutório, apresentando o projeto que gerou o referido produto, assim como conceitos sobre lixo eletrônico e curiosidades sobre a produção mundial de resíduos tecnológicos. Avançando, traz um aprofundamento, trazendo dados sobre os elementos químicos presentes nos eletrônicos e seus riscos para a saúde e meio ambiente.

Como já apontado, a proposta dos passatempos foi propor atividades lúdicas que colaborassem com a fixação dos conteúdos abordados e posterior contribuição para a aprendizagem pelos leitores. Como um exemplo, a Figura 2 apresenta um dos passatempos presentes na cartilha.

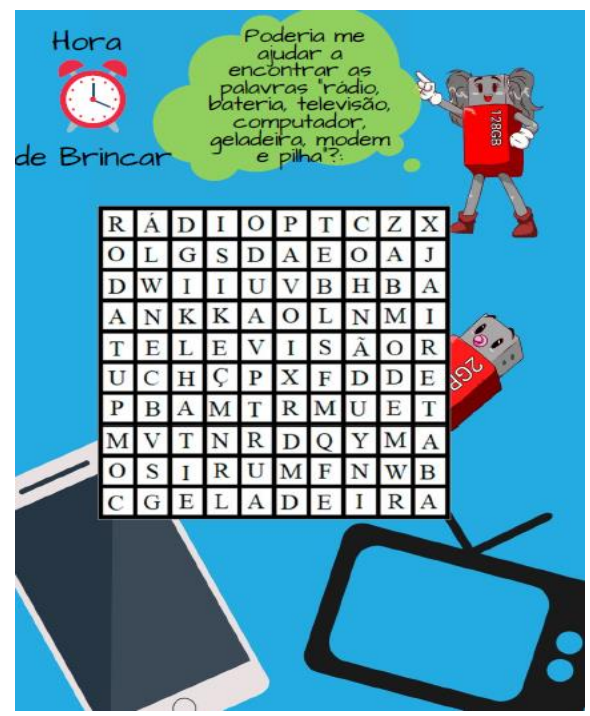

Figura 2: Um dos passatempos propostos.
O material também traz um infográfico mostrando o ciclo de vida de um eletrônico, desde a sua fabricação até a sua possibilidade de reaproveitamento. O objetivo foi mostrar ao leitor que o ciclo não precisa terminar, necessariamente, no descarte, e sim enfatizar o caráter cíclico das tecnologias e, assim, motivar o reaproveitamento e até mesmo a postergação do descarte. A Figura 3 ilustra o infográfico apresentado.

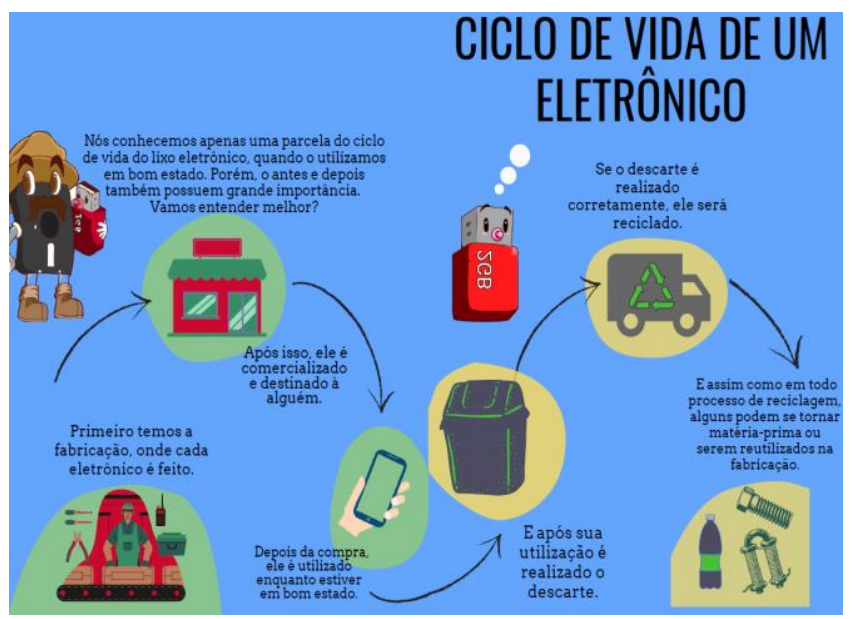

Figura 3: Informações sobre ciclo de vida dos eletrônicos.

Além do conteúdo conceitual, informações relacionadas à saúde e os passatempos, a cartilha visou disseminar iniciativas de coleta e reaproveitamento de lixo eletrônico, listando a legislação sobre o tema, além de campanhas locais realizadas pelo poder público, em parceria com o IFMS Campus Nova Andradina, como ilustrado pela Figura 4. Também foram disponibilizados links externos para aprofundamento sobre tais ações.

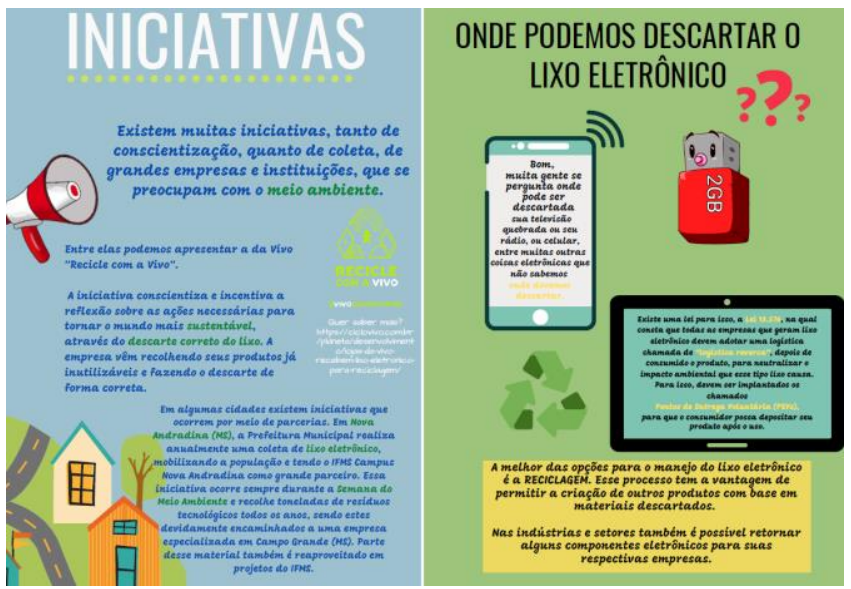

Figura 4: Informações sobre iniciativas de reaproveitamento.

O percurso da leitura proposta, então, visa levar o autor a iniciar com um contato mais conceitual, aprofundando questões mais científicas e culminando com o acesso a informações referentes a possibilidades de engajamento em ações ambientais que visam a 
melhor destinação aos resíduos sólidos tecnológicos. Tudo isso intercalado com os passatempos, trazendo um reforço à leitura de modo lúdico e educativo.

\section{RESULTADOS ALCANÇADOS}

O material produzido foi disponibilizado a docentes de Química e Biologia do IFMS Campus Nova Andradina e da Rede Estadual de Ensino localizadas na cidade. No IFMS o material foi enviado às turmas de $1^{\circ}$ e $2^{\circ}$ ano dos cursos Técnicos Integrados em Agropecuária e Informática, e na Rede Estadual para estudantes dos anos iniciais do Ensino Médio Regular. Por conta da suspensão das aulas nas redes devido à pandemia Covid-19, o material foi enviado pelos docentes aos seus estudantes por meio dos contatos de e-mail e grupos de aplicativos de mensagens.

Neste primeiro momento, a distribuição ainda não atingiu todas as escolas da cidade, e nem estudantes de Ensino Fundamental, porém foi possível obter os primeiros dados provenientes do feedback dado por parte dos estudantes atendidos. Para isso, um questionário foi disponibilizado para manifestação espontânea dos leitores. Até a redação deste artigo, quarenta (40) leitores responderam ao instrumento.

Do total de respondentes, $75 \%$ aumentaram o seu interesse em saber sobre lixo eletrônico após o contato com a cartilha. Todos os respondentes $(100 \%)$ denotaram um aprendizado de todo o conteúdo apresentado e o entendimento de que, após a leitura do material, hoje possuem mais conhecimento sobre o assunto.

Os passatempos apresentados agradaram a todos os respondentes, que destacaram a facilidade de entendimento das propostas e o cruzamento com o conteúdo trabalhado, denotando um elemento de contribuição para a sua aprendizagem. Alguns ainda se mostraram surpresos por não conseguirem, de imediato, resolver todas as atividades, despertando o interesse em buscar leituras complementares para sua resolução completa.

Quando questionados sobre o que mais chamou a atenção no material, as respostas (abertas) apontam, principalmente, o fato de, além de apresentar os perigos do lixo eletrônico, a cartilha mostrar os componentes químicos e os males à saúde humana. Parte dos respondentes também se mostrou surpresa com as estatísticas referentes à produção anual de lixo eletrônico no mundo.

Visando a melhoria do material para novas versões, os leitores foram questionados sobre quais elementos merecem uma atenção maior e quais conteúdos podem ser trazidos futuramente. Do total de respondentes, $50 \%$ destacaram a importância de se trazer mais iniciativas de reaproveitamento existentes e as possibilidades de melhor destino aos resíduos produzidos por cada cidadão. Isso denota a importância desse conteúdo na cartilha. Parte dos leitores (25\%) também julga importante trazer mais curiosidades, assim como os passatempos. Para $25 \%$ dos respondentes, a diagramação pode ser melhorada, deixando o material ainda mais apresentável e atraente, segundo os relatos.

Quanto aos conteúdos, as respostas indicaram, prioritariamente, a necessidade de um aprofundamento do processo de reciclagem, apresentando as informações de modo mais gráfico e intuitivo.
Também foi indicado o anseio por mais informações sobre como as empresas tratam seus próprios resíduos e quais as iniciativas implementadas pela indústria tecnológica aos seus consumidores.

Por fim, quando questionados se indicariam o material lido para amigos e parentes, $100 \%$ dos respondentes indicaram que sim, denotando a aceitação do material pelo público-alvo do projeto.

Vale ressaltar que os resultados aqui apresentados são iniciais, visto que pretende-se, futuramente, fazer um trabalho mais abrangente, incluindo os anos finais do Ensino Fundamental, assim como a obtenção de um feedback dos docentes colaboradores, o que permitirá uma análise mais pedagógica do material e seu posterior aprofundamento para versões futuras.

\section{CONSIDERAÇÕES FINAIS}

Espera-se como resultados deste projeto que haja uma melhor compreensão das características do lixo eletrônico, sua produção e seus impactos ao meio ambiente, de modo que os usuários se conscientizem sobre práticas que possam reduzir tais impactos, desde o consumo até o reaproveitamento de componentes outrora inutilizados. A região de Nova Andradina carece de iniciativas socioambientais e a disponibilização do material aqui proposto apresenta-se como uma contribuição educativa.

Dessa maneira, com a criação da cartilha, houve a pretensão de causar um impacto positivo nas pessoas quanto à história da evolução tecnológica e seu rápido desenvolvimento, além de mostrar alternativas mais baratas e com menor impacto ambiental, quando se fala de renovação de equipamentos.

Para os estudantes envolvidos na execução do projeto houve um enriquecimento quanto aos conceitos necessários para o bom desempenho profissional como técnicos em Informática, além de desencadear neles o espírito científico e extensionista.

\section{AGRADECIMENTOS}

À Fundação de Apoio ao Desenvolvimento do Ensino, Ciência e Tecnologia do Estado de Mato Grosso do Sul (FUNDECT) e ao Conselho Nacional de Desenvolvimento Científico e Tecnológico (CNPq), pelo fomento ao projeto por meio do Programa de Iniciação Científica Júnior (PIBIC-Jr) - CHAMADA FUNDECT/CNPq/SED-MS - Nº6/2019 - PIBIC-Jr-MS.

\section{REFERÊNCIAS}

[1] Fruet, H. 2000. Lixo eletrônico. In Revista Istoé, n. 1587.

[2] Tanaue, A. C. B.; Bezerra, D.; Cavalheiro, L. e Pisano, L. C. 2015. Lixo eletrônico: agravos a saúde e ao meio ambiente. In Ensaios e Ciência: Ciências Biológicas, Agrárias e da Saúde, v. 19, n. 3, p. 130-134.

[3] Forti, V.; Baldé, C. P.; Kuehr, R. e Bel, G. 2020. The Global E-waste Monitor Quantities, flows, and the circular economy potential 2020.

[4] Calixto, B. 2012. Seus eletroeletrônicos também podem ser reciclados. In Revista Istoé - Eletrônicos (online).

[5] Monteiro, M. S. e Romito, P. R. 2012. TI Verde - Implementação de Práticas Sustentáveis em Empresa de Tecnologia da Informação. In Simpósio de Excelência em Gestão e Tecnologia, 9., Resende/RJ, p. 1-10.

[6] Ferreira, J. M. B.e Ferreira, A C. 2008. A Sociedade da Informação e o Desafio da Sucata Eletrônica. In Revista de Ciências Exatas e Tecnologia, v. 03, n. 3, p. 157-170.

[7] Pinto, T. M. C. e Savoine, M. M. 2011. Estudo Sobre TI Verde e sua Aplicabilidade em Araguaína. In Revista Cientifica do ITPAC, v. 04, n. 02, p. 415 . 